\title{
Structure-Soil-Structure Interaction effects on the dynamic response of piled structures under obliquely-incident seismic shear waves.*
}

\author{
Guillermo M. Álamo, Luis A. Padrón, Juan J. Aznárez, Orlando Maeso
}

Insituto Universitario de Sistemas Inteligentes y Aplicaciones Numéricas en Ingeniería (IUSIANI), Universidad de Las Palmas de Gran Canaria, Edificio Central del Parque Científico y Tecnológico, Campus Universitario de Tafira 35017 Las Palmas de Gran Canaria, Spain guillermo.alamo / luis.padron / juanjose.aznarez / orlando.maeso @ulpgc.es

\begin{abstract}
This work studies the structure-soil-structure interaction (SSSI) effects on the dynamic response of nearby piled structures under obliquely-incident shear waves. For this purpose, a three-dimensional, frequency-domain, coupled boundary element - finite element (BEM-FEM) model is used to analyse the response of a configuration of three buildings aligned parallel to the horizontal component of the wave propagation direction. The SSSI effects are studied in terms of the maximum shear force at the base of the structures both in frequency- and time-domains. The results are presented in a set of graphs so that the magnitude of the interaction effects in configurations of buildings with similar vibration properties depending on the distance between them and the angle of incidence can be easily estimated. These results show a high influence of the wave type and angle of incidence on the interaction effects, not always corresponding the worst-case scenario with the commonly assumed hypothesis of vertical incidence. It is found that for configurations of non-slender structures, the SSSI effects can significantly amplify or reduce the single building maximum response depending on the separation between structures and excitation.
\end{abstract}

Keywords: structure-soil-structure interaction, seismic response, angle of incidence, BEM-FEM coupling, pile foundations

\section{Introduction}

When studying the seismic response of civil constructions, the structure is usually considered alone on the ground without any other near structure. However, this situation

\footnotetext{
*Draft of the paper published in SOIL DYNAMICS AND EARTHQUAKE ENGINEERING, 78, 142153 (2015). DOI: http://dx.doi.org/10.1016/j.soildyn.2015.07.013. Accepted: 25 July 2015
} 
rarely happens in modern urban areas. When excited by a seismic input, the vibration of one structure propagates through the soil and reaches the nearby buildings, modifying their dynamic response. This effect is referred to as structure-soil-structure interaction (SSSI) and can either magnify or attenuate the structural response of a building.

To the best knowledge of the authors, some of the first works that proposed models for the quantification of SSSI effects are [1] (that studied the dynamic response of near nuclear reactors on rigid circular bases) and [2, 3] (that studied the bidimensional interaction problem of buildings under antiplane shear waves). The results published in these papers proved that, in some cases, the interaction effects modify the structural response in a way that cannot be neglected. Following these pioneering works, several authors have tackled the SSSI problem through different methodologies, such as analytical solutions, numerical models and experimental tests. As a full literature review is out of the scope of this research, the authors want to refer the interested reader to the work of Lou et al. [4], where a complete survey of the SSSI studies can be found. A related problem that has been addressed more recently [5-7] is that of the site-city interaction (SCI). These works showed that ground motion is affected by the presence of large groups of buildings and that the response of each structure varies significantly from one to another, in such a way that some of them may suffer high damages, while others will remain unaffected.

In most of those works, when studying the interaction phenomena, the seismic excitation is considered to propagate vertically through the terrain. However, Wong and Trifunac [3] studied the interaction between two buildings under SH waves with different angles of propagation. Their results showed different building responses depending on this incident angle. Up to the authors knowledge, no other works have studied the relation between the angle of incidence and the SSSI, being this an aspect of the interaction problem that demands further research.

On the other hand, the response of pile foundations under waves with a generic angle of propagation had been studied in terms of kinematic interaction factors in different works [e.g. 8-10]. One of the most extensive results were obtained by Kaynia and Novak [1] for different pile groups configurations under oblique volumetric waves and Rayleigh waves. These results demonstrate the importance of the angle of incidence in the dynamic response of this type of foundation.

The objective of this work is to include the assumption of a generic angle of incidence in the SSSI study in order to analyse the influence of this parameter on the interaction effects. For this purpose, the dynamic response of a group of three piled structures subjected to planar oblique shear waves is obtained through a direct approach by using a previously developed BEM-FEM model [12, 13]. Coupled BEM-FEM methodologies has been previously chosen by several authors to treat the SSSI problem. Wang and Schmid [14] and Lehmann and Antes [15] used different BEM-FEM models to study the interaction between near structures on embedded foundations. Wang and Schmid [14] used harmonic forces on the structures as the excitation, while Lehmann and Antes [15] placed the load on the ground surface. In their recent work, Clouteau et al. [16] compared the results obtained by their BEM-FEM model with an experimental test using mock-up structures built on unmade ground performed by the Nuclear Power Electric Corporation (NUPEC) in Japan. The numerical results were in good agreement with the experimental ones. 


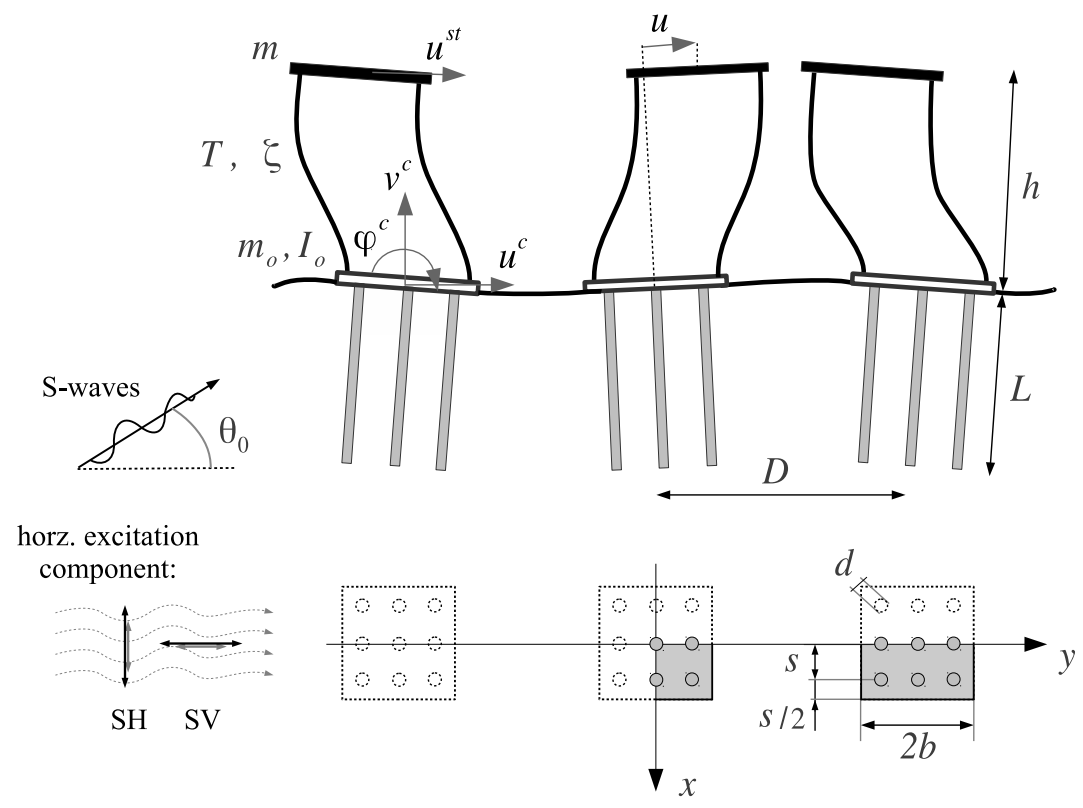

Figure 1: Geometry, symmetry and degrees of freedom of the problem

Along this study, the interaction effects will be quantified by comparing the maximum response of the group buildings with the maximum response of a single structure on the ground. The results, both in frequency- and time-domains, are presented in a set of graphs that allows to evaluate the importance of the SSSI depending on the configuration and excitation. Besides, these results can be understood as correcting factors that can be applied to simplified models in order to include the effects of near constructions and a non-vertical incidence.

\section{Problem statement}

\subsection{Problem definition}

A configuration of three one-storey shear structures founded on $3 \times 3$ fixed-head pile groups embedded on a viscoelastic half-space is studied. The three buildings are aligned with the horizontal component of the wave propagation direction in order to investigate the shielding effect produced by the presence of neighbour foundations in the wave course. The problem is sketched in Fig. 1. The geometric properties of pile groups are defined by: length $L$ and diameter $d$ of piles, center-to-center distance between adjacent piles $s$ and foundation halfwidth $b$. The parameters that define the structures are: cap mass $m_{0}$ and moment of inertia $I_{0}$, fixed-base fundamental period $T$ and structural damping ratio $\zeta$, structure effective height $h$ and mass $m$ and distance between adjacent structures $D$. As the buildings are modelled as one-storey structures, the values of $h, m$ and $\zeta$ can represent either the height, mass and damping of one-storey constructions or the ones equivalent to one particular mode of multi-mode structures.

The dynamic response of each structure is represented by eight degrees of freedom 


$\begin{array}{lcc}\text { piles aspect ratio: } & L / d & 15 \\ \text { piles separation ratio: } & s / d & 5 \\ \text { pile-soil modulus ratio: } & E_{p} / E_{s} & 100 \\ \text { soil-pile density ratio: } & \rho_{s} / \rho_{p} & 0.7 \\ \text { soil Poisson ratio: } & \nu_{s} & 0.4 \\ \text { soil hysteretic damping ratio: } & \beta & 0.05 \\ \text { complex soil shear modulus: } & \mu=\operatorname{Re}[\mu](1+2 \mathrm{i} \beta)\end{array}$

Table 1: Soil and piles properties.

$\begin{array}{lcc}\text { structural aspect ratios: } & h / b & 2,3,5 \\ \text { structure-soil stiffness ratio [17]: } & 1 / \sigma=h /\left(T c_{s}\right) & 0.25 \\ \text { structure-soil mass ratio: } & \delta=m /\left(4 \rho_{s} b^{2} h\right) & 0.15 \\ \text { foundation-structure mass ratio: } & m_{0} / m & 0.25 \\ \text { foundation moment of inertia: } & I_{0} /\left(m h^{2}\right) & 0.05 \\ \text { structure hysteretic damping ratio: } & \zeta & 0.05 \\ \text { complex structural stiffness: } & k=\operatorname{Re}[k](1+2 \mathrm{i} \zeta)\end{array}$

Table 2: Soil-structure system properties.

corresponding to: horizontal translations of vibrating mass $u^{\text {st }}$ and foundation $u^{c}$ along the $x$ and $y$ axes, one vertical displacement $v^{c}$, two rocking motions $\varphi^{c}$ around horizontal axes and one rotational motion $\phi^{c}$ around the vertical axis. As the buildings are modelled as shear structures, the vertical, rocking and rotational motions of cap and floor slab are assumed to be coincident.

The site is assumed to be excited by obliquely-incident SH or SV waves producing horizontal displacements perpendicular or parallel to the alignment of the structures, and with a direction of incidence with respect to the horizontal defined by the angle $\theta_{0}$ (see Fig. 1).

\subsection{Problem parameters}

The mechanical dimensionless properties of the pile-soil system are those presented in Table 1, which can represent reinforced concrete piles foundations in sandy soils. On the other hand, the constants defining the properties of the soil-structure system are listed in Table 2, where $c_{s}$ is the soil shear wave velocity. These values were chosen in order to be representative for typical constructions and have been used in previous studies [1720]. In order to see the dynamic behaviour of the selected soil-structure system, Fig. 2 shows the ratio between the flexible-base $(\tilde{T})$ and the fixed-base $(T)$ fundamental periods depending on $\sigma$, for the properties defined above. These curves were obtained following the methodology presented in [21]. Notice that as the value of $1 / \sigma$ tends to zero (soft structures or stiff soils) the behaviour of the building tends to the fixed-base system one, so no soil-structure interaction is observed. The chosen value of $1 / \sigma=0.25$ allows SSSI effects to be significant enough while being in the range of realistic building properties.

The distance between adjacent buildings will be expressed in terms of the soil wave 


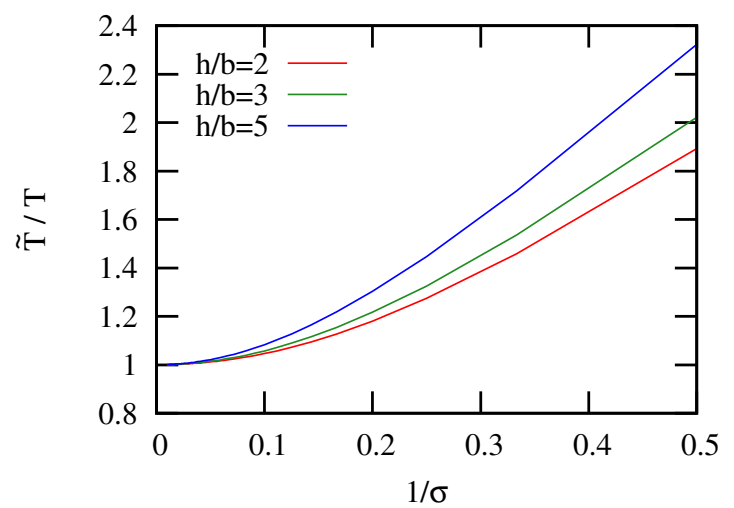

Figure 2: Soil-structure interaction effects measurement for the soil-structure system adopted in this study.

length at the soil-structure fundamental frequency: $D \propto \lambda=c_{s} \tilde{T}$.

\section{Methodology}

In order to obtain the seismic response of the system, a previously developed threedimensional frequency-domain BEM-FEM model [12, 13] is used where the soil is modelled by boundary elements as a homogeneous, semi-infinite, isotropic, linear, viscoelastic medium, while the piles and piers are modelled as linear Bernoulli beams by finite elements. Such a model was used in [20] to study the SSSI effects in piled structures under vertical seismic excitation and was also used in [22] to study the effect of the incidence angle and type of wave in the dynamic response of pile groups and a single piled structure.

The analysis of the SSSI effects is made in frequency-domain through the study of the shear forces at the base of the structure. These forces are obtained by using the Frequency Response Function (FRF) $Q=\operatorname{Abs}\left[\Omega^{2} u / \omega^{2} u_{f f}\right]$ being $\Omega=2 \pi / T$ the structure fundamental frequency, $u_{f f}$ the horizontal free-field displacement and $u$ the structure lateral deformation obtained by subtracting the cap rigid body contribution from the horizontal displacement of the slab $\left(u=u^{s t}-u^{c}-h \varphi^{c}\right)$. This frequency response function represents the ratio of the shear force at the base of the structure to the effective seismic force [23].

The excitation consists of a planar wavefront that propagates through the halfspace with a generic direction contained in the $y z$ plane defined by the angle of propagation $\theta_{0}$. When a wave reaches the soil surface, other waves must be reflected in order to satisfy the free-surface boundary condition (Fig. 3). The harmonic displacements of the domain points are obtained by adding the contribution of each wave:

$$
\mathbf{u}=\sum_{j=1}^{n} \mathbf{d}^{(j)} A^{j} e^{-i k_{j}\left(\mathbf{s}^{(j)} \cdot \mathbf{r}\right)}
$$

where $\mathbf{u}$ is the vector of displacements of the point located at the position defined by $\mathbf{r}$, $n$ is the total number of waves (incident + reflected), $\mathbf{d}^{(j)}$ is the vector containing the 


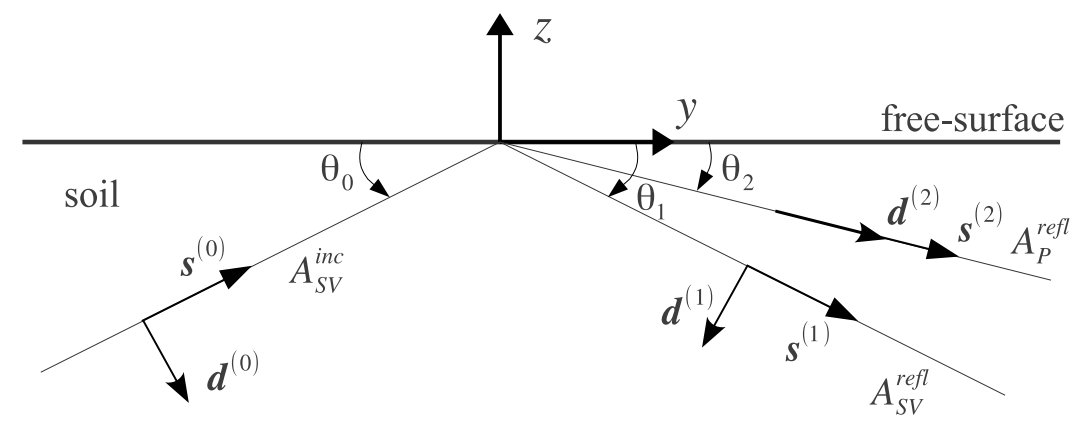

Figure 3: Incident and reflected waves for obliquely-incident SV waves.

direction cosines of the displacements produced by the $j$-th wave, $A^{j}$ is the amplitude of the $j$-th wave, $k_{j}$ is the wave number of the $j$-th wave and $\mathbf{s}^{(j)}$ is the propagation vector of the $j$-th wave.

Two different incident waves are considered in this work: SH and SV. The first one produces displacements in the direction $x$ and reflects another $\mathrm{SH}$ wave. The incident SV wave (Fig. 3), producing displacements perpendicular to the direction of propagation in the $y z$ plane, reflects another $\mathrm{SV}$ wave and a $\mathrm{P}$ wave. If the angle of incidence is smaller than a critical angle (with the soil Poisson ratio used: $\theta_{c r}=65.9^{\circ}$ ) a surface wave is reflected instead of the $\mathrm{P}$ wave. The vectors and amplitude expressions for each wave depends on the angle of incidence and are detailed in [22]. No material damping is considered in the computation of the incident wave-field.

\section{Results}

The SSSI effects under study will be presented in terms of the relative difference between the maximum shear force per effective seismic excitation of the building in the group $\left(Q_{\max }\right)$ and the one experienced by a single building with the same characteristics $\left(Q_{\max }^{r e f}\right)$ :

$$
\Delta Q_{\max }=\frac{Q_{\max }-Q_{\max }^{r e f}}{Q_{\max }^{r e f}}
$$

Positive values of $\Delta Q_{\max }$ mean that the forces the building suffers as a part of the group are higher than the ones that it would present if it were alone; negative values mean that the SSSI effects lead to a reduction in the maximum efforts at the base of the structure. These variations, illustrated in Fig. 4, depend on the excitation type and propagation angle, the distance between adjacent buildings and the position of the building in the group.

The maximum values of each FRF are compared regardless of the frequency at which they take place. Variations in the peak frequency will be also obtained in relative terms as:

$$
\Delta \omega_{p}=\frac{\omega_{p}-\omega_{p}^{r e f}}{\omega_{p}^{r e f}}
$$




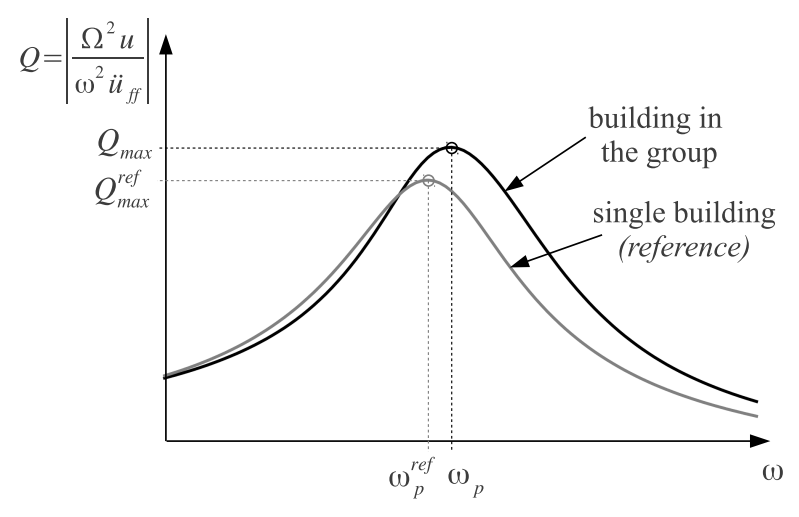

Figure 4: Qualitative representation of changes in the shear force frequency response function due to the interaction effects.

The different configurations studied in this work are summarized in Table 3. Note that for cases $\mathrm{A}, \mathrm{B}$ and $\mathrm{C}$ the buildings in the group are compared with the single building under the same excitation (type and angle), while in cases D and E the reference single building is excited by a vertical incidence.

\subsection{Influence of distance on SSSI phenomena}

This section focuses on illustrating how the response of each building and the interactions between them strongly depend on the separation distance. Thus, general conclusions cannot be drawn from the results of just one single configuration.

Fig. 5 presents the maximum shear force variation as a function of the separation distance for the case A which corresponds to three identical buildings with an aspect ratio $h / b=2$. The separation distance goes from $0.2 \lambda$ to $\lambda$ with a $\lambda / 40$ step. Each row corresponds to a different building position in the group and different angles of incidence are represented by different lines. Representative incidence angles of $60^{\circ}, 75^{\circ}$ and $90^{\circ}$ are studied. Structure-soil-structure interaction effects are apparent in Fig. 5 as the variations in the maximum shear force are different from zero, which means that the maximum responses of the buildings in the group differ from the maximum response of the single building system. Note also that, even for vertical incidence $\left(\theta_{0}=90^{\circ}\right)$, the responses of the lateral buildings and the central structure do not coincide due to SSSI [20].

The worst-case scenario changes with the structure position, the wave type and its angle of propagation. Depending on the distance of separation, the interaction effects produces the highest variations at different angles of incidence, not always corresponding the worst situation to the vertical incidence hypothesis.

For this reason, responses will be computed for five representative separation distances $(D=\lambda / 4, \lambda / 3, \lambda / 2,3 \lambda / 4$ and $\lambda)$ identified in Fig. 5 . 


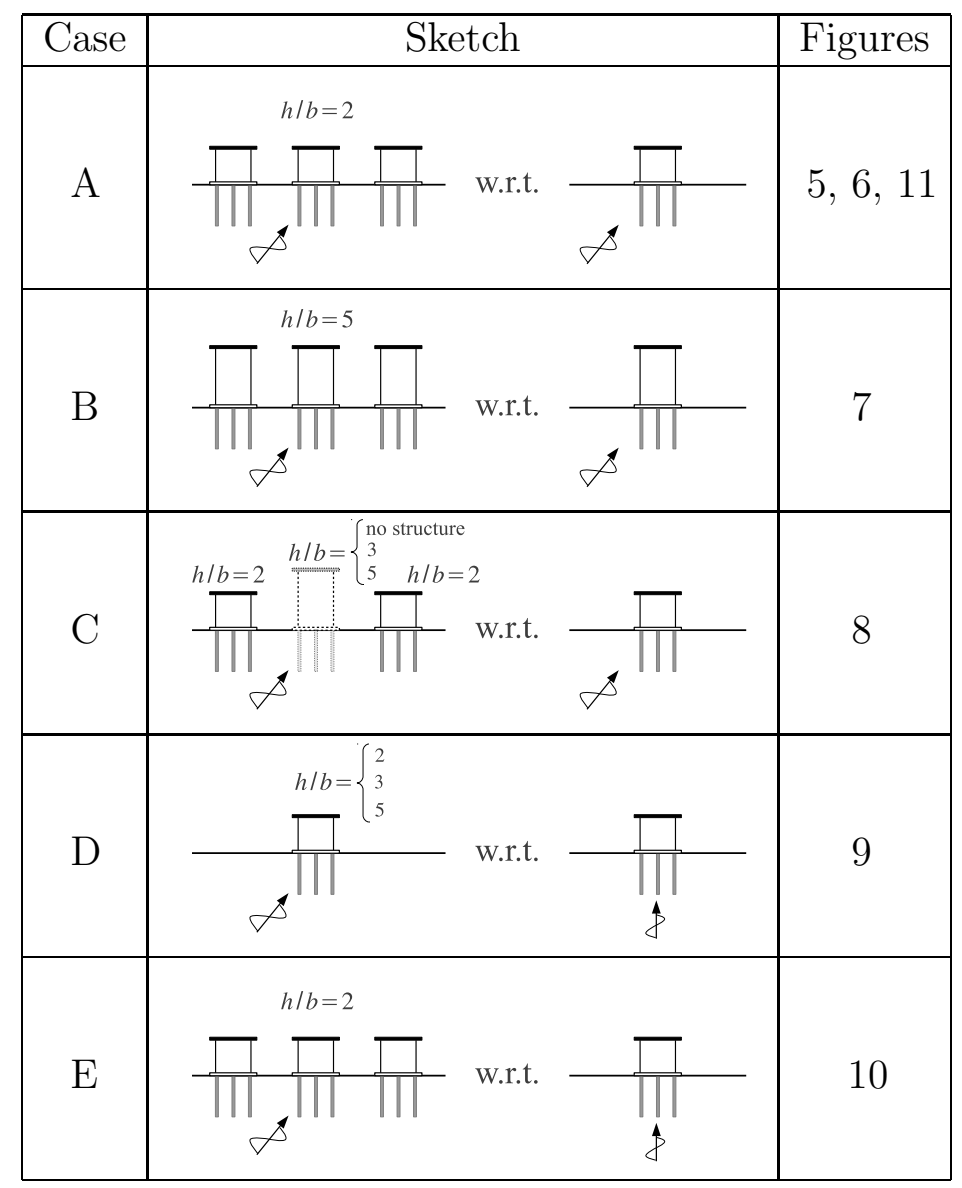

Table 3: Cases under study. 


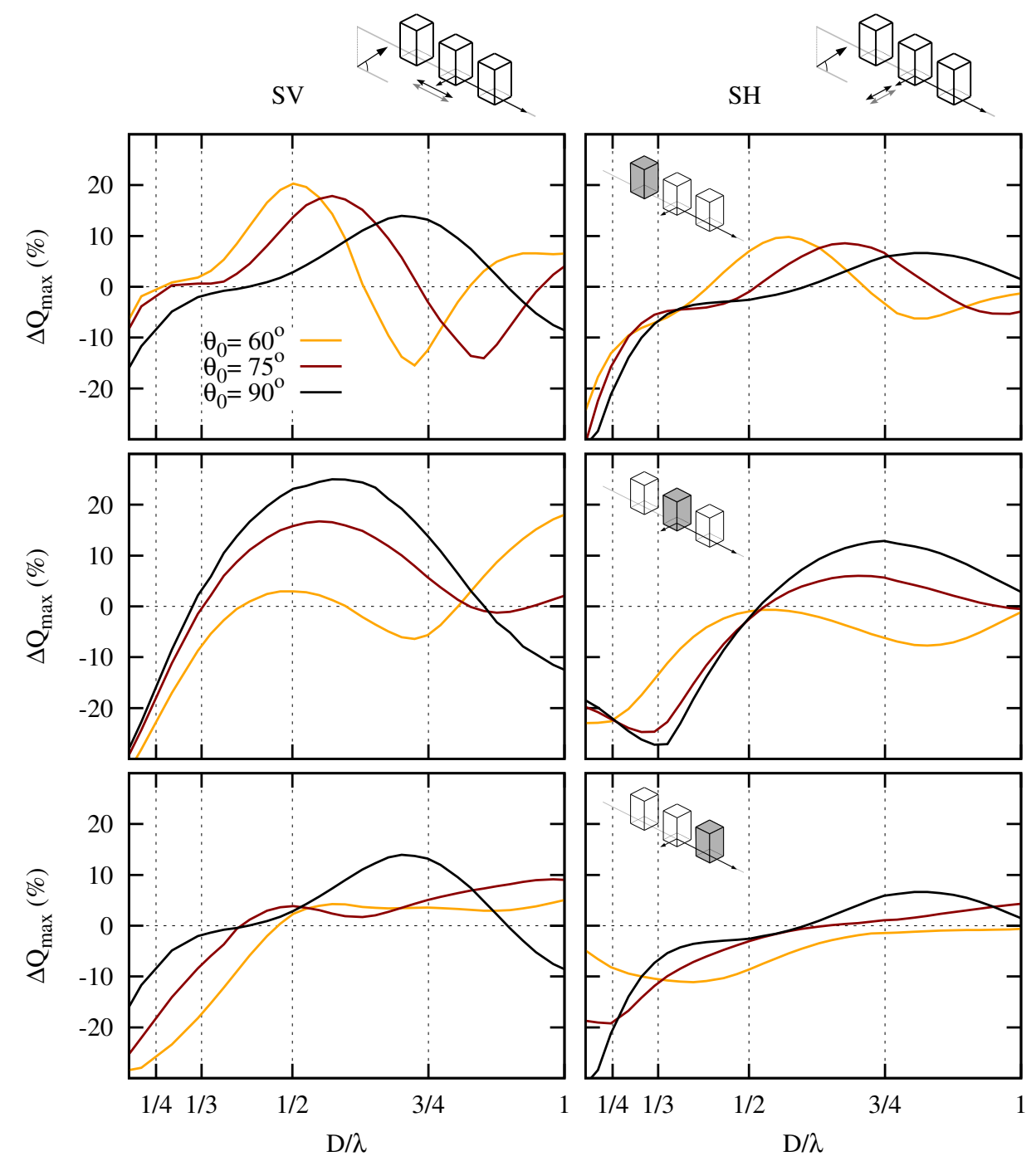

Figure 5: Case A. Variations in the maximum shear force as a function of the separation distance. Structural aspect ratio $h / b=2$. 


\subsection{Influence of angle of incidence}

Fig. 6 presents the variations of the maximum shear forces in case A as a function of the angle of incidence for the set of distances defined above. The different separations between adjacent buildings are presented in rows, while the two wave types are presented in columns. The response of every structure in the group is plotted by different line colors according to the diagram at the top of the figure.

Depending on the studied configuration and excitation, the magnitude of the variations in the maximum shear force changes: great reductions, close to $30 \%$, take place for both wave types at small separation distances; while the highest increments of the maximum efforts are produced for SV waves and longer distances, reaching values over $20 \%$.

The angle of incidence has a clear influence on the interaction effects. This influence is more evident for the lateral buildings: considering vertical incidence, the responses of the first and last structures coincide due to the problem symmetry; but, as the angle of incidence becomes smaller, their curves follow different trends depending on the building position due to three factors: the spatial character of the excitation, the shielding effect and the interaction effects between the buildings.

The shielding effect can be observed between the buildings in the group for SV waves and small separation distances. This effect produces that the maximum shear force variation of the first building augments as the angle of incidence becomes smaller, while the variation of the two last buildings is reduced. This effect loses importance as the distance between buildings increases. For SH waves no shielding effect can be appreciated.

For the selected distances, the worst-case scenario corresponds to a separation between buildings of $D=\lambda / 2$ and SV waves, for which the buildings present higher efforts than the single structure system. However, the importance of considering a non-vertical incidence is highlighted for separations of $D=\lambda$. At this separation and SV waves, the SSSI effects cause reductions of the maximum shear forces for vertical incidence but increase these maximum efforts if the incidence is oblique.

\subsection{Influence of the structural aspect ratio.}

Fig. 7 presents the variations of the maximum shear force at the base of the structures for buildings with an aspect ratio of $h / b=5$ (case B). For this building typology, the variations of the maximum efforts are significantly smaller than the ones studied before (maximum variations are around $\pm 5 \%$ ). However, the comparison against Fig. 6] shows that the overall trends are very similar with independence of the aspect ratio, even if magnitudes are different. Only some differences can be seen between the results of the two structural aspect ratios for small separations.

\subsection{Influence of the differences among the structures within the group}

Fig. 8 shows the maximum shear force variations for the study case $\mathrm{C}$, where the scenarios with different central structures are analysed: small variations between structures (using a $h / b=3$ ratio for the central building), strong variations between structures (with a central structural aspect ratio $h / b=5$ ) and no central structure (and no foundation). 


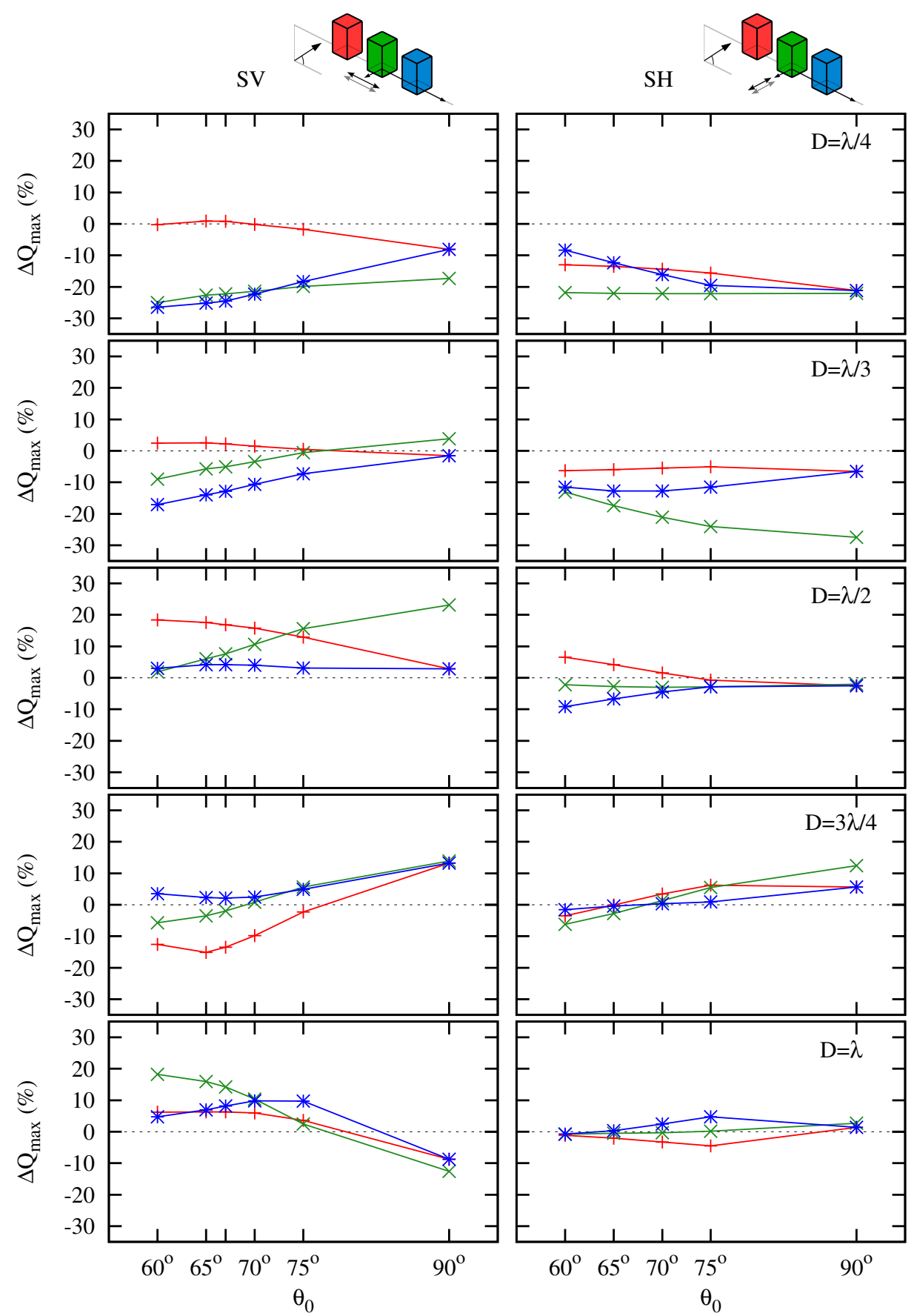

Figure 6: Case A. Variations in the maximum shear force as a function of the angle of incidence and wave type. Structural aspect ratio $h / b=2$. 




Figure 7: Case B. Variations in the maximum shear force as a function of the angle of incidence and wave type. Structural aspect ratio $h / b=5$. 
The distances between buildings are expressed in terms of a $\lambda$ corresponding to the lateral structures $(h / b=2)$.

The maximum responses of lateral buildings differ from the ones observed in the configuration of three identical structures (Fig. 6). Besides, the maximum responses of lateral buildings are practically independent of the central structure characteristics, providing this central structure is different from the lateral ones. The interaction between two structures occurs at frequencies near the fundamental ones (i.e., the variations in the response of structure A due to the presence of structure B take place at the surroundings of fundamental frequency of structure B and vice versa). For this reason, only the interaction between structures with close fundamental periods will be appreciated in this study focused on maximum response. This explains why Fig. 8 shows almost no differences between the three studied configurations. However, for SV waves, the interaction between the two lateral buildings is slightly modified by the presence of the central structure and its foundation for small distances due to the nature of the SV waves (displacements and propagation in the same plane) that makes them more sensitive to buried obstacles or structures than the $\mathrm{SH}$ waves.

On the other hand, maximum efforts in central buildings are not significantly affected by the presence of lateral structures with different vibrational properties. The highest variations occur for small distances and principally for SV waves.

\subsection{Influence of non-vertical incidence}

The previous results were presented using as reference value the response of the single structure system subjected to the same excitation (wave type and angle of incidence). This was made with the intention of isolating the SSSI effects. However, the response of a single structure is usually obtained under vertical incidence instead of under oblique incidence. It would therefore be interesting to be able to refer all results to the case of vertical incidence in order to include simultaneously the influence of SSSI and oblique incidence. For this reason, this section analyses the case in which response under vertical incidence are taken as reference results.

First, Fig. 9 illustrates the influence of a non-vertical incidence on the maximum efforts of a single structure (case D) by presenting the relative difference between the maximum shear force at each angle of incidence with respect to the maximum response at vertical incidence.

For SH waves, the maximum shear force in a single structure system is almost independent on the incident angle. On the contrary, significant variations can be seen for SV waves. For angles of incidence below the critical angle great reductions in the maximum shear force are produced. For such angles, the horizontal displacement and rocking of the cap become out of phase so the inertial forces that the storey suffers are attenuated. The maximum shear force amplification is produced at the critical angle and decays as the angle of incidence increases. Comparing the different structural aspect ratios, higher values are produced for smaller ratios.

Now, Fig. 10 presents the relative differences between the maximum shear forces in the buildings in a group under obliquely-incident waves with respect to that of a single building system under vertically-incident waves (case E). This figure illustrates how such relative differences are a combination of those due to SSSI effects (shown in Fig. 6] for case 




Figure 8: Case C. Variations in the maximum shear force as a function of the angle of incidence and wave type. Lateral structures aspect ratio $h / b=2$, central structure aspect ratio $h / b=3$ and 5 . 


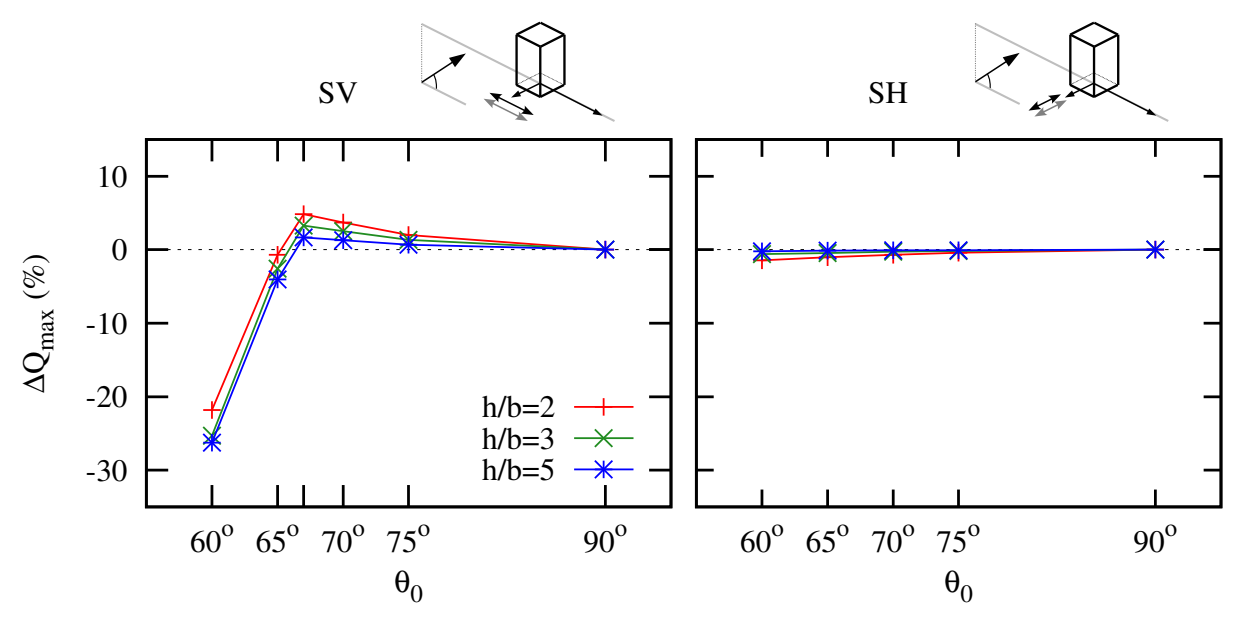

Figure 9: Case D. Variations in the maximum shear force as a function of the angle of incidence and wave type for a single building.

A) and those due to oblique incidence (as shown in Fig 9 for case D). From its definition and through simple mathematical operations, this relationship can be written as:

$$
\Delta Q_{\max }^{(E)}=\Delta Q_{\max }^{(A)}+\Delta Q_{\max }^{(D)}\left(\Delta Q_{\max }^{(A)}+1\right)
$$

By using this expression one can quantify how much the maximum response of the single building under vertical incidence is modified by both the interaction and oblique incidence effects for any of the studied configurations.

\subsection{Peak frequency variations}

Fig. 11 presents the variations of the frequency at which the maximum response value takes place for case A, showing that this peak frequency depends on the building position, separation distance, wave type and its angle of incidence. Such variations are due to the fact that the response of the buildings in the group is determined by the superposition of several vibration modes whose contribution changes with the angle of incidence because of the spatial character of the excitation. Those different vibration modes present their fundamental frequencies at near (but not identical) values, reason why the position of the maximum total response moves.

The obtained results show that the interaction effects can either increase or reduce the value of the frequency at which the maximum response takes place. The highest variations occur for the central building and small separation distances, reaching values over $\pm 4 \%$. The peak frequency variations of structures with higher aspect ratio are significantly smaller. Results for the $h / b=5$ structural typology were also obtained but are not shown as they present negligible maximum frequency variations (with values always below $\pm 0.25 \%$ ). 




Figure 10: Case E. Variations in the maximum shear force as a function of the angle of incidence and wave type. Structural aspect ratio $h / b=2$. 




Figure 11: Case A. Variations in the peak frequency as a function of the angle of incidence and wave type. Structural aspect ratio $h / b=2$. 


\begin{tabular}{lll}
\hline \multicolumn{1}{c}{ Soil } & \multicolumn{1}{c}{ Piles } & \multicolumn{1}{c}{ Structures } \\
\hline$c_{s}=237 \mathrm{~m} / \mathrm{s}$ & $E_{p}=2.76 \cdot 10^{10} \mathrm{~Pa}$ & $T=0.2 \mathrm{~s}$ \\
$\rho_{s}=1750 \mathrm{~kg} / \mathrm{m}^{3}$ & $\rho_{p}=2500 \mathrm{~kg} / \mathrm{m}^{3}$ & $m=3.8 \cdot 10^{5} \mathrm{~kg}$ \\
$\nu_{s}=0.4$ & $d=0.8 \mathrm{~m}$ & $h=12 \mathrm{~m}$ \\
$\beta=0.05$ & $L=12 \mathrm{~m}$ & $\zeta=0.05$
\end{tabular}

Table 4: Soil, piles and structures dimensional properties.

\subsection{Maximum temporal response variations}

In the previous sections, results in the frequency-domain were obtained. In this section, the most relevant results will be presented also in terms of time-domain variables in order to check whether the conclusions drawn above can be extrapolated to the response of the structures under seismic events. For that purpose, expression (22) is used again to compute the maximum effort variations but considering now that the studied variable corresponds to the maximum value of the time response of each building in the group or the reference single building. In order to obtain the time-history forces at the base of the structures, the standard frequency-domain method [23] is used. Note that, as the seismic input is included to obtain the time response, in this section the comparison is made in terms of the maximum shear force $\left(V_{\max }\right)$ rather than the maximum shear force per effective seismic force $\left(Q_{\max }\right)$.

The seismic excitations are synthetic accelerograms generated by SIMQKE [24] to be compatibles with the type 1 response spectrum for ground type $\mathrm{C}$ presented in Part 1 of Eurocode 8 [25]. These accelerograms correspond to the free-field horizontal acceleration at the position of the central structure. The fundamental period of the structures $T=$ $T_{B}=0.2 \mathrm{~s}$ is chosen in order to match the one where the maximum response occurs. The rest of the problem dimensional properties are obtained by setting the modulus of elasticity of piles $E_{p}=2.76 \cdot 10^{10} \mathrm{~Pa}$ and density of piles $\rho_{p}=2500 \mathrm{~kg} / \mathrm{m}^{3}$ (reinforced concrete piles) and are summarized in Table 4.

A set of 20 independent accelerograms is used in order to handle the high variability of the results depending on the seismic input. The mean value of the maximum effort variations is used as representative value and their variability is expressed through the standard deviation.

Fig. 12 shows the variations of the maximum temporal shear forces at the base of the structures for case A. These are the time-domain results corresponding to the frequencydomain ones presented in Fig. 6. Comparing both figures, one can see that variations in the maximum efforts follow the same trends with the angle of incidence and separation distance. However, the time variations, with maximum values around 10\%, are smaller than the harmonic ones, presenting maximum values above $20 \%$. The value of the maximum effort of the time response varies depending on the accelerogram used as excitation. These deviations from the mean value (which can reach values around $\pm 5 \%$ in some configurations) highlight the importance of considering different input data in this type of studies. 


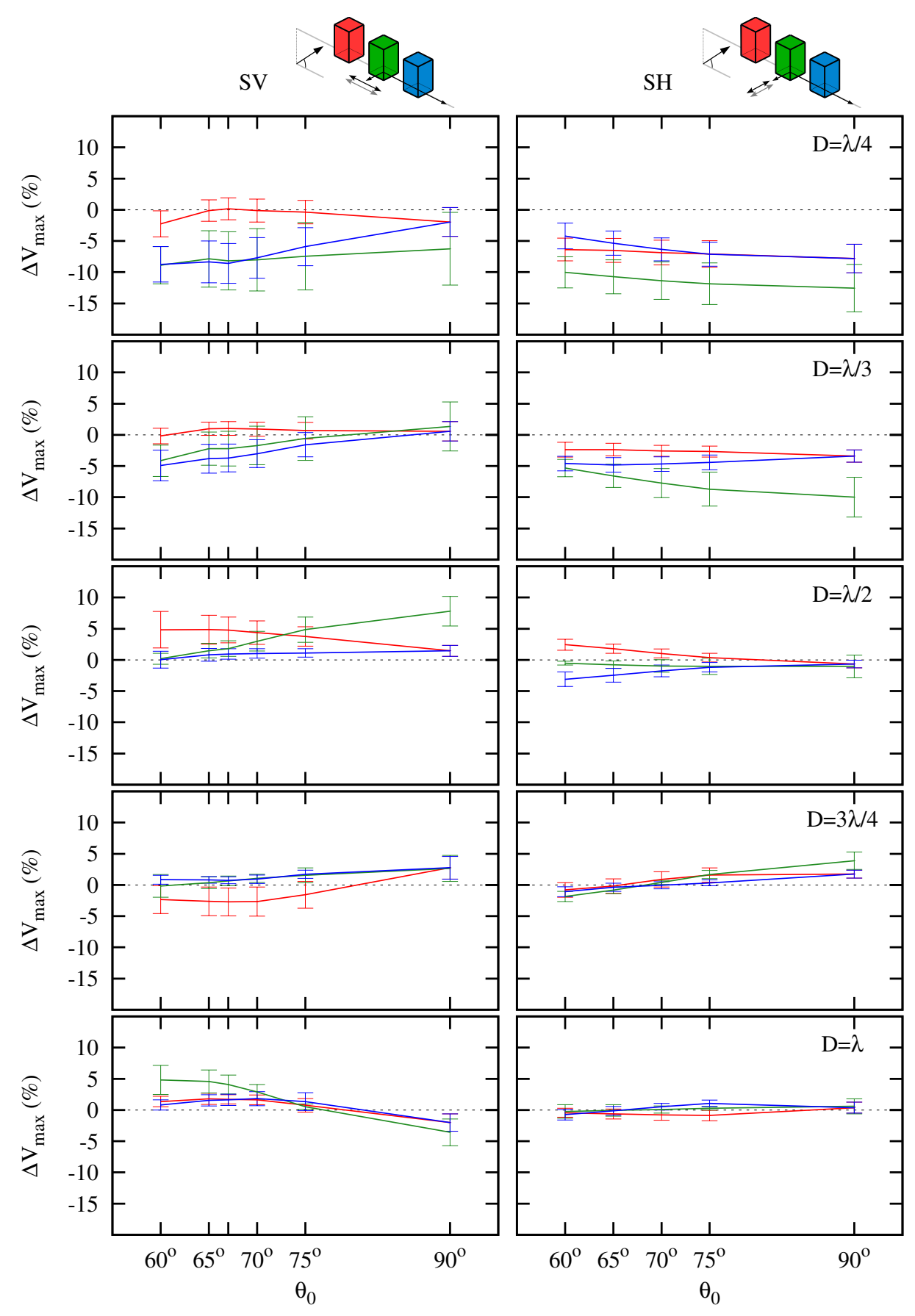

Figure 12: Case A. Variations in the maximum temporal shear force as a function of the angle of incidence and wave type. Mean value and standard deviation from 20 independent accelerograms. Structural aspect ratio $h / b=2$. 


\section{Conclusions}

In this work, a configuration of three piled buildings under obliquely-incident shear waves is studied in order to investigate the influence of the wave type and its angle of propagation on the structure-soil-structure interaction among them. The SSSI effects are measured by comparing the maximum responses of the buildings in the group with the maximum response of the soil-structure system formed by a single structure with identical characteristics. The buildings responses, in terms of the shear force at the base of the structure per effective seismic force, are obtained by a frequency-domain BEM-FEM model assuming linear behaviour of soil, piles and structures.

The obtained results show that the type of wave and its angle of incidence have a great influence on the SSSI effects, not always corresponding the worst situation to the vertical incidence hypothesis. These effects are strongly related to the structure on-soil fundamental frequency as they have the same behaviour independent on the structural aspect ratio if the distance between buildings is expressed in terms of the soil wave length at this frequency. Moreover, significant interactions are only produced between buildings with similar fundamental periods. The interaction effects on the maximum shear forces are more important for buildings with smaller structural aspect ratios.

The SV waves are more affected by the presence of obstacles under the terrain. For small distances between buildings and oblique incidence, a shielding effect can be observed for these incident waves. The shielding effect causes the first structure to increase its maximum shear force variation as the angle of incidence becomes smaller, while the two last buildings variations are reduced.

When comparing with the single structure system under vertical incidence, the effects of an oblique incidence have to be considered. This principally affects the SV waves, causing high reductions in the maximum efforts for angles below the critical one and a peak in the response at its surroundings. For SH waves, the response of the single building is almost independent on the angle of incidence.

The maximum forces at the base of the buildings in the group occur at different frequencies depending on the excitation and building position. These variations in the single structure fundamental frequency are produced due to the variable contribution of the different vibration modes that form the total response. The peak frequency variations only are significant for small aspect ratios.

For small separations between buildings high reductions in their maximum shear forces are produced. On the other hand, the highest variations depend on the wave type, angle of incidence and building position. In general, the worst configurations correspond to separation distances between $0.5 \lambda$ and $0.7 \lambda$ (reaching increments in the maximum shear efforts over $20 \%$ for small structural aspect ratios).

Finally, when studying the buildings time response, the structure fundamental period has been chosen to coincide with the one where the excitation presents more energy. With this properties, the variations of the maximum time efforts follow the same trends as the harmonic results but with smaller values, which means that the same conclusions reached in the frequency-domain can be applied to the time response. 


\section{Acknowledgements}

This work was supported by the Ministerio de Economía y Competitividad (MINECO) of Spain and FEDER through research Project BIA2010-21399-C02-01 and also by the Agencia Canaria de Investigación, Innovación y Sociedad de la Información (ACIISI) of the Government of the Canary Islands and FEDER through research Project ProID20100224. In addition, G.M. Álamo is a recipient of a fellowship from the Program of predoctoral fellowships of the University of Las Palmas de Gran Canaria. The authors are grateful for these supports.

\section{References}

[1] Lee, T.H., Wesley, D.A.. Soil-structure interaction of nuclear reactor structures considering through-soil coupling between adjacent structures. Nucl Eng Des 1973;24(3):374-387. doi:10.1016/0029-5493(73)90007-1.

[2] Luco, J.E., Contesse, L.. Dynamic structure-soil-structure interaction. Bull Seismol Soc Am 1973;63(4):1289-1303.

[3] Wong, H.L., Trifunac, M.D.. Two-dimensional, antiplane, building-soil-building interaction for two or more buildings and for incident plane SH waves. Bull Seismol Soc Am 1975;65(6):1863-1885.

[4] Lou, M., Wang, H., Chen, X., Zhai, Y.. Structure-soil-structure interaction: Literature review. Soil Dyn Earthq Eng 2011;31(12):1724-1731. doi:10.1016/j.soildyn.2011.07.008.

[5] Clouteau, D., Aubry, D.. Modifications of the ground motion in dense urban areas. J Comput Acoust 2001;9(4):1659-1675. doi:10.1142/S0218396X01001509.

[6] Tsogka, C., Wirgin, A.. Simulation of seismic response in an idealized city. Soil Dyn Earthq Eng 2003;23(5):391-402. doi:10.1016/S0267-7261(03)00017-4.

[7] Kham, M., Semblat, J.F., Bard, P.Y., Dangla, P.. Seismic site-city interaction: Main governing phenomena through simplified numerical models. Bull Seismol Soc Am 2006;96(5):1934-1951. doi:10.1785/0120050143.

[8] Mamoon, S.M., Banerjee, P.K.. Response of piles and pile groups to travelling SHwaves. Earthq Eng Struct Dyn 1990;19(4):597-610. doi:10.1002/eqe.4290190410.

[9] Mamoon, S., Ahmad, S.. Seismic response of piles to obliquely incident SH, SV, and P waves. J Geotech Eng 1990;116(2):186-204. doi:10.1061/(ASCE)0733-9410(1990)116:2(186).

[10] Makris, N., Badoni, D.. Seismic response of pile groups under obliqueshear and rayleigh waves. Earthq Eng Struct Dyn 1995;24(4):517-532. doi:10.1002/eqe.4290240405. 
[11] Kaynia, A.M., Novak, M.. Response of pile foundations to rayleigh waves and obliquely incident body waves. Earthq Eng Struct Dyn 1992;21(4):303-318. doi:10.1002/eqe.4290210403.

[12] Padrón, L.A., Aznárez, J.J., Maeso, O.. BEM-FEM coupling model for the dynamic analysis of piles and pile groups. Eng Anal Bound Elem 2007;31(6):473484. doi:10.1016/j.enganabound.2006.11.001.

[13] Padrón, L.A., Aznárez, J.J., Maeso, O.. 3-D boundary element-finite element method for the dynamic analysis of piled buildings. Eng Anal Bound Elem 2011;35(3):465 - 477. doi:10.1016/j.enganabound.2010.09.006.

[14] Wang, S., Schmid, G.. Dynamic structure-soil-structure interaction by FEM and BEM. Comput Mech 1992;9(5):347-357. doi:10.1007/BF00370014.

[15] Lehmann, L., Antes, H.. Dynamic structure - soil - structure interaction applying the symmetric galerkin boundary element method (SGBEM). Mech Res Commun 2001;28(3):297-304. doi:10.1016/S0093-6413(01)00177-X.

[16] Clouteau, D., Broc, D., Devésa, G., Guyonvarh, V., Massin, P.. Calculation methods of structure-soil-structure interaction (3SI) for embedded buildings: Application to NUPEC tests. Soil Dyn Earthq Eng 2012;32(1):129-142. doi:10.1016/j.soildyn.2011.08.005.

[17] Veletsos, A.S., Meek, J.W.. Dynamic behaviour of building-foundation systems. Earthq Eng Struct Dyn 1974;3(2):121-138. doi:10.1002/eqe.4290030203.

[18] Bielak, J.. Dynamic behaviour of structures with embedded foundations. Earthq Eng Struct Dyn 1974;3(3):259-274. doi:10.1002/eqe.4290030305.

[19] Avilés, J., Pérez-Rocha, L.E.. Evaluation of interaction effects on the system period and the system damping due to foundation embedment and layer depth. Soil Dyn Earthq Eng 1996;15(1):11-27. doi:10.1016/0267-7261(95)00035-6.

[20] Padrón, L.A., Aznárez, J.J., Maeso, O.. Dynamic structure-soil-structure interaction between nearby piled buildings under seismic excitation by BEM-FEM model. Soil Dyn Earthq Eng 2009;29(6):1084-1096. doi:10.1016/j.soildyn.2009.01.001.

[21] Medina, C., Aznárez, J.J., Padrón, L.A., Maeso, O.. Effects of soil-structure interaction on the dynamic properties and seismic response of piled structures. Soil Dyn Earthq Eng 2013;53(0):160-175. doi:10.1016/j.soildyn.2013.07.004.

[22] Zarzalejos, J.M., Aznárez, J.J., Padrón, L.A., Maeso, O.. Influences of type of wave and angle of incidence on seismic bending moments in pile foundations. Earthq Eng Struct Dyn 2014;43(1):41-59. doi:10.1002/eqe.2330.

[23] Chopra, A.K.. Dynamic of structures. Theory and applications to earthquake engineering. NJ: Prentice-Hall; 2001. 
[24] Vanmarcke, E.H.. SIMQKE: A program for artificial motion generation. Tech. Rep.; Massachusetts Institute of Technology, Cambridge, MA; 1976.

[25] Eurocode 8: Design of structures for earthquake resistance. Part 1: General rules, seismic actions and rules for buildings. Standard EN-1998-1, CEN/TC 250. 2003. 